\title{
V.
}

\section{Die Einkleidung des platonischen Parmenides. Von}

Dr. phil. J. Eberz.

Erwägt man die aus der Diogenes-Angabe von der Blütezeit des Parmenides sich ergebende chronologische Unmöglichkeit einer Begegnung des Eleaten mit Sokrates, ferner die unhistorische Zeichnung der Personen des platonischen Dialoges, jenes schon die Alten als willkürliche Erfindung befremdende Liebesverhältnis des Parmenides und Zenon, den ganz ungeschichtlichen Charakter dieses unklarèn und hilflosen, wegen überstürzter Hypothesen, an die der Sohn des Sophroniskos nie dachte, abgekanzelten Sokrates, und das von keiner Tradition erwähnenswert befundene bedeutende philosophische Talent des Tyrannen Aristoteles, endlich gar den ganzen Inhalt des Werkes, die Deduktion der notwendigen Setzung einer xotvwvía von Ideen zur Begründung der Möglichkeit der Erfahrung ${ }^{1}$ ) als Antwort auf ein Material von Angriffsgeschützen, wie es erst Aristoteles aufgefahren hatte, so möchte man keinen Augenblick anstehen, allen kleinen Rechenkünsten zum Trotz die unser Gespräch motivierende Begegnung mit einigen Alten wie Athenaeus ${ }^{2}$ ) und Macrobius ${ }^{3}$ ) and Neueren wie Steinhart und Campbell ${ }^{4}$ ) für eine willkürliche Fiktion Platons zu erklären.

Bedenkt man hingegen auf der anderen Seite, daß, wenn überhaupt ein Dialog Platons Gelegenheitsschrift ist, dann sicherlich

1) Natorp, Platos Ideenlehre p. 215-271.

?) XI, $505 \mathrm{f}$.

3) Sat. I, 1.

4) Classical Review 1596 (X) p. 135.

Archiv fur Geschichte der Philosophie. XX. 1. 
unser Gespräch, als das problematischste unter allen platonischen, ganz bestiumten äußeren Umständen entwachsen sein muß, aus der Einsicht, in welche erst der Zweck des ganzen seltsamen Werkes begriffen werden kann, so daß es undenkbar erscheint, Platon habe durch eine fingierte Motivierung die wirkliche verdrängen können, un unbegreiflicherweise das Verständnis des Ganzen zu erschweren oder gar zu vereiteln, da doch das fundamentale Problem von solcher Wichtigkeit für ihn ist; bedenkt man ferner, wie hier so natürlich und ungekünstelt die Untersuchung aus der Situation hervorgeht, die zahlreichen intimen, ein wirkliches Erlebnis verhalten andeutenden Striche der Zeichnung, die Sicherheit, mit der besonders die Altersangabe des Eleaten vorgetragen wird, so möchte man wiederum mit Schleiermacher nicht zögern, in der einleitenden Erzählung historische Wahrheit zu erblicken.

Wenn man bei diesem Widerspruch nicht skeptisch stehen bleiben will, so wird man von selbst auf die ihn versöhnende Synthese getrieben, daß jene Motivierung zugleich Dichtung und Wahrheit enthält, d. h. Personen, Situation und Gespräch unbedingt historisch, aber die Namen dieser Personen aus irgendeinem Grunde fingiert sind. Diesem Postulate gibt folgende Erwägung seine bestimmte Richtung. Da in dem Dialoge das vitale Problem des Platonismus, das Verhältnis der Ideen zu den Dingen, behandelt ist, und zwar in musterhaft schulmäßig-nüchternem Ton und der knappen Prägnanz einer festgewordenen wissenschaftlichen Schulsprache, da diese Erörterung in einem engen Kreise von Platonikern stattfindet, dessen Geschlossenheit zweimal ${ }^{5}$ ) geflissentlich betont wird, derart, daß nicht zu zweifeln ist, daß wir „in dem ganzen Dialog, von der ersten bis zur letzten Zeile, auf dem Boden der Akademie stehen": ${ }^{6}$ ) so müssen wir in den Personen bedeutendere Mitglieder dieser Schule vermuten, welche bei der Wichtigkeit, die der Sitzung beigelegt wird, gewiß keine alltägliche Gelegenheit zusammengeführt hat. Es fragt sich also, ob wir imstande sind, die hinter jenen Namen stehenden realen Träger des Gespräches

5) Parm. 136d; 13 ia.

6) Natorp l. c. 
trotz ihrer Masken zu erkennen, die Situation in .ihrer historischen Bedeutung zu rekonstruieren. und sie als Begründung der spekulativen Untersuchung zu verstehen, so daß durch das Licht, welches auf die Veranlassung derselben fiele, zugleich ihre wahre Absicht außer Zweifel gestellt würde.

Wenn Platon die Wahl des Aristoteles zum Gesprächsgenossen von so seltsamen Gründen wie von dem durch Parmenides scherzhaft vorgeschobenen Bedürfnis des Ausruhens, dem sogleich der unaufhaltsam einer Windsbraut gleich dahinfahrende Dialog. widerspricht oder von der Hoffnung, bei Aristoteles wegen seiner großen Jugend keiner Widerborstigkeit zu begegnen, abhängig zu machen scheint, so soll solche problematische Begründung stutzig machen und den nachdenklich gewordenen Leser erkennen lassen, daß der Philosoph auf die Einführung dieses Gesprächsgenossen mehr aufmerksam machen als ihre wahren Gründe mitteilen will, daß er aber besonders wichtige Motive für die Wahl einer derart hervorgehobenen Persönlichkeit hatte, wenn er sie auch, sei es was uns immer, lieber von dem Leser selbst will erraten lassen. Aristoteles, aus diesem Kreise erlesener Platoniker in dem positiven Hauptteile der einzige Mitunterredner des Parmenides, muß jedenfalls zu dem hier behandelten Problem in nächster innerer Beziehung stehen, d. h. es muß, nach der Art, wie Platon zu adressieren pflegt, diese ganze Lektion nicht weniger für ihn als für den Sokrates bestimmt sein, womit zugleich zu erkennen gegeben ist, daß er ebensowenig wie dieser bisher im Besitz der wahren Ideenlehre gewesen ist, so daß also beide zusammen wegen ihrer bisherigen Stellung zu demselben Problem vorgenommen werden.

Will man nun nicht die Einheit des Dialoges zerreißen, so muß man annehmen, daß beide, wie sie jetzt wegen der gleichen Frage fast wie Schuldige vor höherer Instanz stehen, auch in persönlichen Beziehungen in einer und derselben Affäre, welche in letzter Linie die Veranlassung zu der Sitzung abgegeben hat, gestanden haben, um in verschiedenem Tone gehaltene Aufklärungen über das von beiden gemeinsam irrig behandelte Problem zu empfangen. Dieses Verhältnis aber kann nur dasjenige von Lehrer und Schüler gewesen sein. Jener $135 \mathrm{c}$ dem Sokrates gemachte 


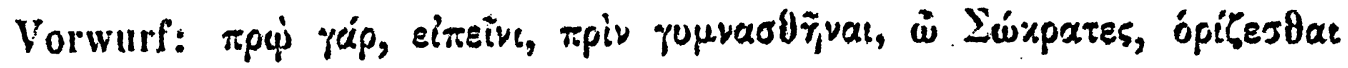

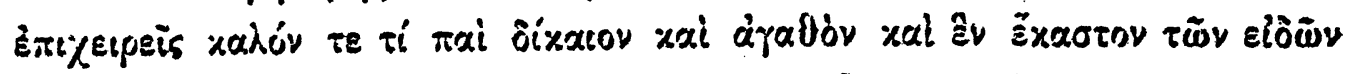
muß nämlich, um als solcher überhaupt Sinn $z \mathfrak{u}$ haben, eine verfrühte, auf mangelhafter dialektischer Vorübung beruhende, sich nach außen wendende Lehrtätigkeit des Mannes treffen, welche eben in jenem ópiceolat bestand, dessen Gegenstand nach dieser Stelle und den vorausgegangenen Aporien die Ideen und ihr Verhïltnis zu den Dingen, gewesen ist: die scharfe Zurechtweisung trifft ihn also ob solcher in der Akademie, vor deren.Elite er steht, ausgeübter Lehrtätigkeit, durch die er die wahre Gestalt der platonischen Lehre zwar in gutem Glauben, aber im Widerspruch mit den Erwartungen, die man in ihn setzen konnte, und der Stelle, welche er bekleidete, kompromittierte. Noch unzweideutiger womöglich wird $135 \mathrm{ab}$ auf diese Tätigkeit des Sokrates angespielt, wo, nachdem die Schwierigkeiten der von ihm vorgetragenen falschen Ideenlehre betont sind, die außerordentlichen intellektuellen und pädagogischen Qualitäten, die für den Lehrer der wahren platonischen Ideenphilosophie erfordert werden, wie etwas, das er nicht besitzt, aber besitzen sollte, ihm zur Ermahnung vorgehalten werdeu. ${ }^{7}$ ) An derselben Stelle wird nun ferner von einem ganz bestimmten "Hörer" (ảxoúwv tís 135a) des Sokrates gesprochen, der durch unwiderleglich scheinende Einwände die Existenz oder wenigstens die Erkennbarkeit der Ideen widerlegt zu haben glaubte: und da in einem ganz parallelen Gedankengange wenige Zeilen später Aristoteles als derjenige genannt wird, welcher den Sokrates in einer Disputation über dasselbe Problem, wie Parmenides, der Zeuge derselben war, andeutet, in die Enge trieb, ${ }^{8}$ ) als offenbar von jenem.

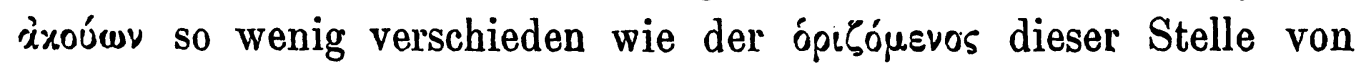
dem vorher 135 a erwähnten, so ist damit Aristoteles deutlich genug. als der Schüler des Sokrates gekennzeichnet. Als solcher hat er: demnach nicht nur die allerdings auch von Platon desavouierte. Ideenlehre des Sokrates bekämpft, sondern, wie der Bericht 135a, die ihm dort gegebene Zurechtweisung, welche analog der dem

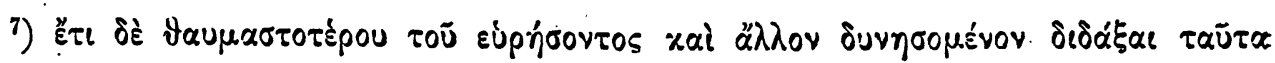

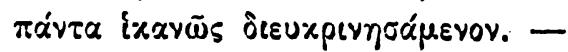

8). $135 \mathrm{~d}$. 
Sokrates erteilten die hohen Anforderungen, die ein Schüler des Platonismus erfüllen muß, betont, und nicht zum wenigsten endlich das ganze ihn belehrende Gespräch mit Parmenides verbürgen, in jener die Ideenlehre überhaupt gedacht widerlegt zu haben. Beide haben mithin, wie sie jetzt gemeinsam vor den Vertretern der Akademie stehen, auch als derselben angehörig gemeinsam geirrt, aber nach ganz verschiedenen Richtungen hin, indem der Lehrer die Transzendenz der Ideen verfocht, die Schüler die Ideen überhaupt negierten; allein gegen den Irrtum des in höherem Grade verantwortlichen Lehrers zeigt sich Platon unnachsichtlicher als gegen den noch sehr jungen und offenbar auch noch nicht lange der Schule verbundenen Aristoteles.

Diesem ersten im Verhältnis von Lehrer und Schüler stehenden, in Athen ansässigen Paare tritt ein zweites gegenüber, welches am Panathenaeenfeste aus dem griechischen Westen angekommen ist: der fünfundsechzigjährige, Parmenides genannte Philosoph und sein den Namen Zenon führender Lieblingsschüler. Zwischen dem Tag der ;Ankunft und dem der Sitzung muß einige Zeit verstrichen sein, während welcher Parmenides Zeuge jener Disputation des jungen Sokrates und Aristoteles wurde ${ }^{\text {(, }},{ }^{9}$ ) und Pythodoios den Zenon im voraus sein Werk hat vorlesen hören. Beachtung verdient ferner auch des Zenon Bemerkung, daß er, der doch in Begleitung des Parmenides angelangt ist, gleichwohl seit langem von demselben keine philosophischen Auseinandersetzungen mehr gehört habe. ${ }^{10}$ )

Aus irgendeinem einstweilen gleichgültigen Grunde lehrt der Meister diesmal nicht unter dem Namen des Sokrates, sondern als Parmenides, um, von eleatischen Begriffen ausgehend, jenem akademischen Lehrer und dem Schüler desselben die autoritative Darstellung des Verhältnisses der Ideen zueinander und zu der Genesis des Objektes der Vorstellung zu geben. Die Reise nach dem Westen, von welcher er eben zurückgekehrt ist, war seine zweite sizilische. $\mathrm{Z}$ war läßt sich zu dieser Einsicht die Altersangabe nicht verwerten,

9) тр⿻і́n $135 \mathrm{~d}$.

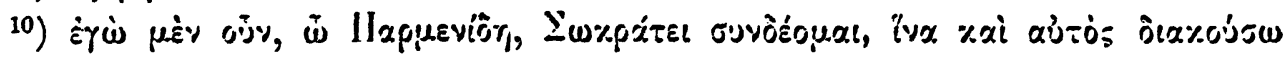

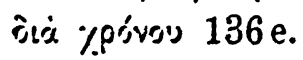


da iiher sein Geburtsjahr Meinungsverschiedenheit herrscht, wohl aher dio Tatsache, dab er von der dritten Reise in einem Jahre mit olympischen Spielen, dem Jahre der durch Helikon vorausgesngten Sonnenfinsternis, 360, zurückkam, während es sich diesmal un das Fest der pentaeterisch in jedem dritten Olympiadenjahr gefeierten großen Panathenäen handelt; ebensowenig kann an die erste Reise gedacht werden, da seine Schule als bereits längere 'Zoit bestehend vorausgesetzt ist. Jene zweite Reise aber trat er $367 \mathrm{an}$, in welchem Jahre bereits vier Monate nach seiner Ankunft Dion verbannt wurde, so daß ihn nun nichts mehr in Syrakus festhalten konnte und er 366, wie allgemein angenommen wird, zurïckkehrte; und dieses Jahr, 0l. 103, 3, war nun gerade ein Jahr mit großen Panathenäen.

Man entrüstete sich im Altertum darüber, daß Platon den

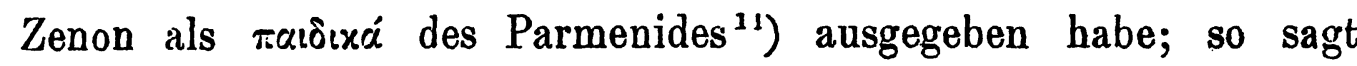

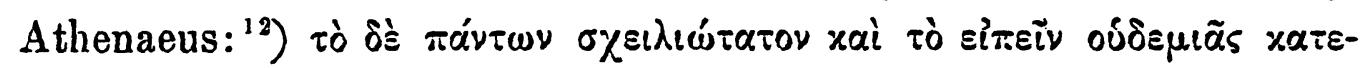

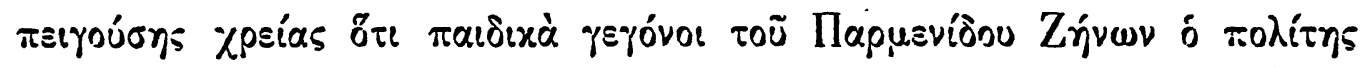

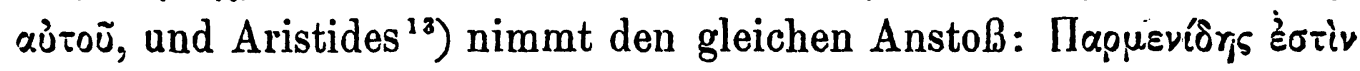

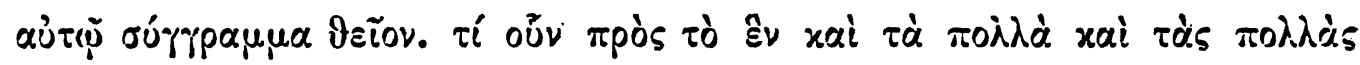

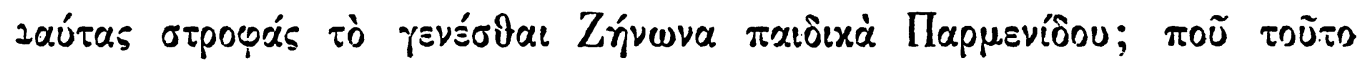

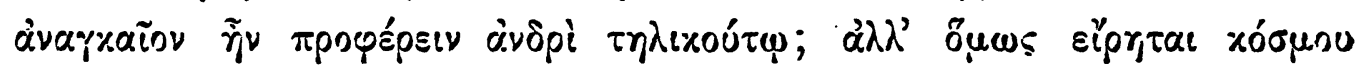

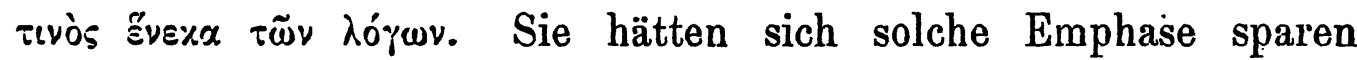
können, wenn sie die Identität des Parmenides und Platon eingesehen hätten; denn auf Grund derselben redet Platon hier von seinem eigenen Liebling, dem Dion; in dessen Begleitung er den Boden der Heimat wieder betritt. Ein neues Zeugnis legt Platon hier für jene Leidenschaft ab, welche für das innere und äußere Leben des Philosophen so bedeutungsvoll gewesen ist, wie nur je die Liebe eines Dante zu einer Beatrice, und welcher zwei so verschiedene Interpreten wie Gomperz ${ }^{14}$ ) und Natorp ${ }^{15}$ ) Platons hohes Lied der Liebe einstimmig zuschreiben, welche den jungen Dionysios mit

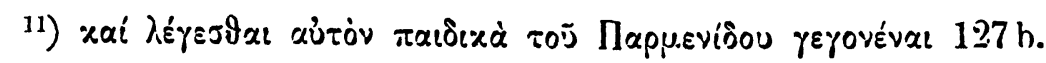

12) XI, $505 \mathrm{f}$.

13) Ad Capit. 326.

14) Griechische Denker II ${ }^{2}$, p. 319; cf. 572.

. ${ }^{15)}$ Natorp l. c. p. $166 \mathrm{~A}$. 
törichter Eifersucht erfüllte und der Platons Epigramm auf den Tod des Freundes das ergreifendste Denkmal gesetzt hat. Da nnn Dion nach seiner Verbannung aus Syrakus seinen Wophnsitz in Korinth ${ }^{16}$ ) nahm, so ist Platon in dieser Stadt wieder mit ihm zusammengetroffen, um von dort aus, ähnlich wie 360 von Olympia, mit ihm gemeinsam die Heimreise anzutreten: so versteht man nun auch jene oben erwähnte Äußerung Dions, den Platon, mit dem er doch eben angekommen ist; seit langem, nämlich nicht mehr seit den wissenschaftlichen Sitzungen bei Dionysios, gehört zu haben. Jetzt aber führt Platon seinen Lieblingsschüler, wie er ihn demonstrativ gegen Dionysios aus Korinth abgeholt hat; ebenso demonstrativ selbst feierlich in den engeren Kreis seiner akademischen Freunde ein, um ihn in Zukunft ganz unter seinem Einflusse zu behalten ${ }^{17}$ ) als den Nächsten unter den Nahen, wenn auch der junge Sokrates ob solcher Bevorzugung, seinen spitzen Worten nach zu urteilen, eifersüchtig scheint: ${ }^{18}$ ) zwischen jenen beiden aber bedarf es des bloßen Blickes, um einander zu verstehen. ${ }^{19}$ ) Den Namen Zenon endlich hat Dion erhalten, weil er dessen Werk mit herübergebracht ${ }^{20}$ ) und der Akademie zum erstenmal die direkte Kenntnis desselben ermöglicht hat; für Platon, welcher der geistigen Verwandtschaft, in der sein Lieblingsschüler zu ihm stand, Ausdruck geben wollte, bot sich dann der Name des Mannes, dessen Lieblingsschüler Zenon gewesen war, ganz von'selbst, der des Parmenides: und weil er zudem gerade in dieser Epoche seines Denkens dem großen Eleaten die fruchtbarsten Anregungen verdankte, so übernahm er diesmal denselben auch mit der gleichen inneren $\mathrm{Be}$ gründung wie sonst den des Meisters Sokrates.

Die problematischste Person des Werkes ist der junge Sokrates, welcher den Athetesen Ü̈berwegs und Schaarschmidts so gute Dienste

16) Nep. Dio. c. 4.

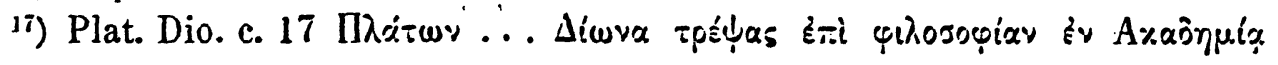
รग)

18) Parm. 12Sa.

19) $130 \mathrm{a}$.

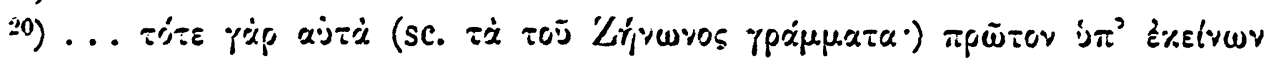

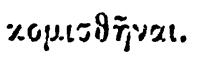


lat leisten müssen. Dabei, in ihm den historischen oder den sogenannten „idealen" Sokrates oder gar den an seiner eigenen Lehre cine so demütigende, ja in diesein Falle geradezu taktlose Kritik übonden l'laton selbst zu erkennen, wird sich mit gutem Gewissen niemand beruhigen können. So viel ist vielmehr gewiß, daß hier keine Phantasiegestalt, sondern eine nach dem Leben gezeichnete wirkliche Persönlichkeit vor uns steht, die jedoch nicht die Ideen als fïr die Genesis des Objektes unerläßliche Faktoren analytisch entdeckt haben kann, sondern zu der jüngeren Generation gehört, welcher jene Lehre bereits als fertiges Dogma überliefert ward, und die nun hintennach herumexperimentiert, um die Denkbarkeit des äußerlich übermittelten, nicht innerlich als Eigentum gefundenen Postulats sich vernunftgemäß zu begründen: mit einem Wort, der junge Sokrates ist ein unfertiges Mitglied der platonischen Akademie. Und diese Persönlichkeit läßt sich nun nach unserer bisherigen Analyse mit Händen greifen. Während Platons Abwesen-

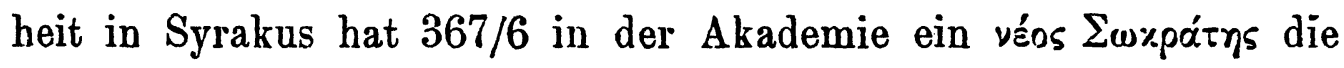
platonische Ideenlehre vorgetragen, d. h. des Meisters eigene Stelle ersetzt. Nun ergibt sich aus der Nàchricht des Laertius Diogenes

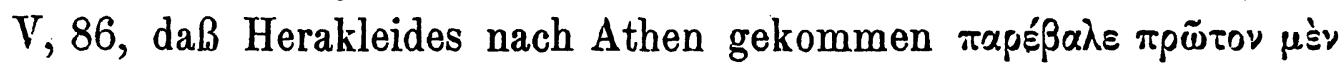
$\Sigma \pi \varepsilon v \sigma i \pi \pi \omega$, notwendig, daß Platon damals nicht in Athen geweilt hat, Speusippos also während einer Reise desselben die Leitung der Akademie in der Hand gehabt haben muß, welche Reise, da er selbst dem Meister auf dessen dritter nach Syrakus folgte, nur dessen zweite Reise nach Sizilien gewesen sein kann, ${ }^{21}$ ) d. h. eben diejenige, von der Platon zu Beginn unseres Dialoges mit Dion zurückkehrt. Folglich muß der in unserem Dialoge als véos $\Sigma \omega$ xpaĩ sein. Haben wir noch nötig, die Berechtigung dieses Pseudonyms näher zu begründen, oder hätte es wegen seiner Durchsichtigkeit sich nicht schon längst verraten müssen, wenn man entschiedener in Platons Werken Dokumente seines eigenen Lebens zu sehen gewagt hätte? Denn was war natürlicher als da $B$, da Platon nun einmal nicht nur in seinen Dialogen sich selbst Sokrates nennt,

21) Fischer, de Speusippi vita p. 26. 
sondern offenbar als Haupt der Akademie in der Schule schlechthin mit diesem Namen bezeichnet wurde, ${ }^{22}$ ) auch sein Neffe, der nun als Stellvertreter seine Rolle zu spielen hatte und wohl schon damals zum dereinstigen Nachfolger bestimmt war, mit dem Amt auch den Namen übernahm, um an Stelle des „alten Sokrates“ als „junger Sokrates“ jenes Lehrtätigkeit auszuüben? Wie fremd das Verständnis der platonischen Ideenlehre in der Tat immer dem Speusippos geblieben ist, beweist zur Genüge der Umstand, daß er die für ihn mit unlösbaren Schwierigkeiten verknüpften Ideen schließlich überhaupt preisgab und die Zahlen an ihre Stelle setzte: und aus der Tatsache, daß er die Transzendenz dieser Zahlen vertrat, ergibt sich hinreichend, in welchem Sinne er vorher die Ideen gesetzt hatte; die dogmatische Natur des Aristoteles aber auch in diesem Falle dem Speusippos gegenüber wie für das Mißverständnis der Lehre Platons verantwortlich zu machen, geht deshalb nicht, weil Aristoteles wohl unterscheidet zwischen jener von Speusippos vertretenen Transzendenz und der von den Pythagoreern behaupteten Immanenz der Zahlen in den Dingen, zwei von ihm ausdrücklich entgegengesetzten Theorien. ${ }^{23}$ ) Die Gleichheit der Lehre beider

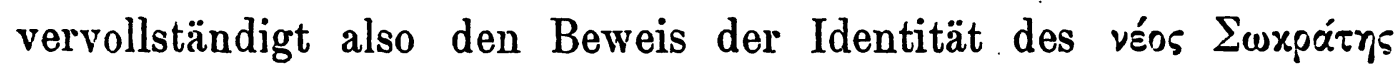
und Speusippos.

Daß der Aristoteles unserer Schrift, welcher sich trotz aller von seinem großen Namensvetter erhobenen, hier vor Platon selbst vorgetragenen, Einwände von der Notwendigkeit der Ideenlehre überzeugen lassen muß, in bestimmter Beziehung zu dem Philosophen, dem in Wirklichkeit die Belehrung zugemünzt werde, zu denken sei, hat bereits Überweg, ${ }^{24}$ ) unter ausdrücklicher Betonung der Verschiedenheit beider Personen, ausgesprochen: nach welcher auch noch von Siebeck ${ }^{25}$ ) geteilten Ansicht er also zu polemischen Zwecken als Mittelsperson ausgewählt wäre, um indirekt, aber deutlich genug, die Zurechtweisung an die richtige Stelle gelangen

2*) Cf. Arist. Pol. II, 6.

23) Die Stellen bei Zeller II, 14, p. 1003, 1.

*4) Untersuchungen p. 182.

25) Platon als Kritiker aristotelischer Ansichten, I Parmenides. Zeitsch. f. Philos. u. philos. Kritik Bd. 107, p. 3. 
zu lassen. Nur die Gesellschaft, in der sich unser Aristoteles belindet, war es, dio jede weitergehende Folgerung, wie verführerisch sie sein mochte, auszuschließen schien: aber gerade die Gesellschaft dieser Männer, deren wahre Namen sich uns nun enthüllt haben, nötigt uns, die Identität jener beiden Personen zu behaupten. schwerlich konnte sich in diesem Kreise, in dem Aristoteles, offenhar, um ihn zur Bescheidenheit und Selbstprüfung zu mahnen, zweimal ausdrücklich als der Jüngste genannt wird, ${ }^{26}$ ) ein noch jüngerer als der Stagirite befinden: stand er doch bei Platọns Rückkehr erst im neunzehnten Lebensjahre. In die Schule Ol. 103, 2 (367) aller Wahrscheinlichkeit nach nicht mehr von Platon selbst, sondern wie Herakleides von Speusippos aufgenommen, hat er jedenfalls bei diesem in des Meisters Abwesenheit, wie es unser Gespräch verlangt, Vorlesungen über die Ideen gehört; und die ernste, aber wohlwollende Belehrung, welche ihm im Gegensatz zu Speusippos zuteil wird, möchte gerade aus der gerechten Erwägung Platons, den Schüler, den er jetzt zum erstenmal gesehen hat, nicht für die Irrtümer des Lehrers verantwortlich machen zu können, wohl aber ihm statt jenes Zerrbildes schleunigst die authentische Fassung der Lehre übermitteln zu müssen, begründet sein. Zugleich scheinen wir den Grund entdeckt zu haben, wieso bei Aristoteles jene falsche Auffassung von den Ideen als überdinglichen Dingen so tiefe Wurzel schlagen konnte, nämlich in jenem wenigstens ein Jahr dauernden Unterrichte des Speusippos gerade in der empfänglichsten Lebensperiode des Schülers, als in der neuen Stadt ein neues äußere und inneres Leben für den Jüngling begann; durch seine dogmatische Sinnesart allen Bemühungen Platons, ihn zum Umlernen zu bestimmen, unzugänglich, konnte er sich, um überhaupt einen Begriff mit diesem Teil der platonischen Philosophie zu verbinden, von der speusippischen Auffassung später nicht mehr losmachen. - Wer nun den Charakter der von Aristoteles in seinen Dialogen gegen die Transzendenz der Ideen geführten Polemik erwägt, jenen aus innerster Notwendigkeit zum Schutz seines eigensten Wesens als gegen eine ihm völlig wider die Natur gehende Theorie erhobenen

. ${ }^{26)}$ Parm. $137 \mathrm{bc}$. 
Widerspruch, von dessen persönlicher Intensität jene von Philoponos überlieferten Worte des Proklos ${ }^{27}$ ) das beredteste Zeugnis sind:

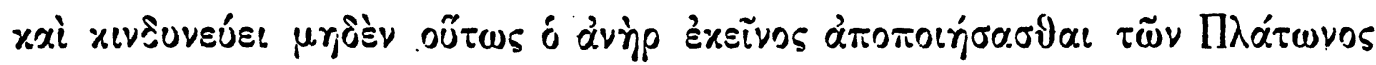

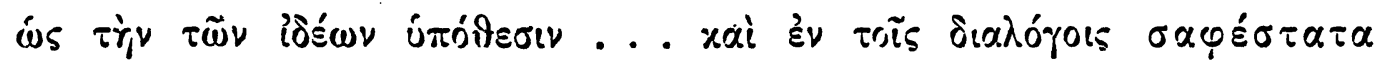

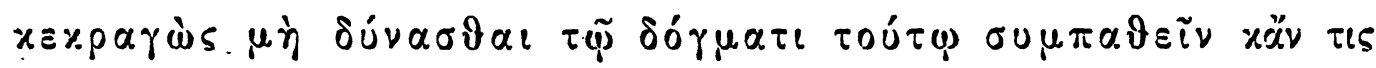

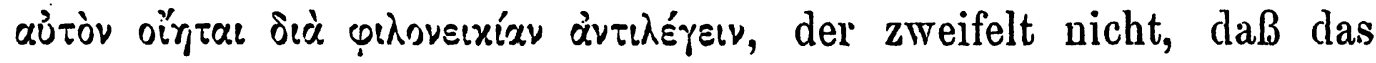
Gesetz, unter welchem diese Natur stand, vom ersten Augenblick an zum instinktiven Widerspruch gegen Speusippos nötigte: und erwägt man ferner, daß die im "Parmenides“ von Platon vorgetragenen Einwände alle oder doch in der wichtigsten Mehrzahl aristotelisches Eigentum sind ${ }^{28}$ ) so hat man in denselben jene von dem Stagiriten in Platons Abwesenheit gegen Speusippos geführten Waffen zu erkennen, in der Haltung des letzteren aber ein treues Abbild seiner damaligen Hilflosigkeit, wie denn von seinem Standpunkt aus an eine Verteidigung auf jene Angriffe nicht zu denken war. Aus der Vollständigkeit und Ordnung dieses Angriffsmaterials möchte man schließen, daß der frühreife Jüngling dasselbe bereits in einem Dialoge niedergelegt hatte; und der Umstand, daß er, auffallend genug, im Gegensatz zu den übrigen Personen seinen wahren Namen behalten hat, kann diese Vermutung nur bestätigen: war es ihm doch eigentümlich, in seinen Dialogen ohne Pseudonym aufzutreten und stets in eigenem Namen seine Sache zu führen. ${ }^{29}$ ) Jetzt, da wir mit den vier am Gespräche sich beteiligenden Nännern der Gesellschaft bekannt geworden sind, erschließt sich mit einemmal die Situation in ihrem bedeutungsvollen Zusammenhange und damit die Bestimmung des ganzen Werkes. Ende Juli oder Anfang August 366 im Alter von 65 Jahren aus Syrakus zurückgekehrt, ${ }^{30}$ ) vernahm Platon durch Speusippos, welcher Rechenschaft von seiner Stellvertretung ablegte, von der kritischen Lage,

2i) Bei Bernays, Die Dialogo des Aristoteles p. 15̄1; cf. die Ausführungen p. $46-48$.

28) Siebeck in der genannten Abhandlung.

29) Bernays 1. c. p. 2 u. 137.

- 30) Trotz Hermodoios ist also das Geburtsjahr Platons nach der eigenen Erklärung des Philosophen das Jahr 431/30. - In der Angabe von Dions Geburtsjahr, den er (c. \&) fünfundfünfzigjährig fallen läßt, ist auch Nepos ungenau, der 366 vierzigjährige Syrakusaner ist $406 / 5$ geboren. 
in wolche dic Ideenphilosophie in der Zwischenzeit durch die Angriffe des jüngst aufgenommenen Aristoteles geraten war. Selbst Zeuge einer Disputation zwischen Speusippos und Aristoteles gewordon, sah er für dio Schule die Gefahr einer Spaltung, die sein lebenswerk bedrohte, so nahe gerückt, daß er es für geboten erachtete, sofort die wichtigsten Mitglieder seiner Akademie zu einer Sitzung zusammenzuberufen, die im Anschuß an die Lektüre der von Dion mitgebrachten zenonischen Schrift die schwebende Frage entscheiden sollte. Zu dieser im Hause des Pythodoios genannten Akademikers abgehaltenen Versammlung kommen getrennt der bisherige Stellvertreter der Schule mit einer Zahl auserlesener Mitglieder derselben ${ }^{31}$ ) und, wohlberechnet zuletzt, erst gegen Schluß der Lektüre, der Meister selbst mit dem Hausherrn und dem jungen Aristoteles, der auf diese Weise feierlich durch Platon selbst in den engeren Kreis der Schule eingeführt wird. Es handelt sich also um mehr als eine bloße „akademische Seminarstunde": es ist einer der historischen Momente im Leben der Akademie, wo Platons Autorität über zwei Schismatiker, von denen jeder Schule gemacht hat, zu Gericht sitzt, um die Reinheit der Lehre wiederherzustellen: es ist eine Art Konzil. Der eine verfocht die Transzendenz der Ideen, der andere leugnete die Ideen schlechtweg: beide Auffassungen sollten durch ihre Vertreter selbst widerlegt und zugleich durch eine Deduktion der Bedingungen der vorgestellten Welt der Dinge die Notwendigkeit des Systems der Ideen positiv dargetan werden. Wir sahen bereits, daß Platons gerechtes Urteil beide nicht gleich behandelt. Dem Speusippos, dem er nicht eine nochmalige beschämende Wiederholung jener durch die aristotelischen Einwände erlittenen Niederlagen ersparen kann, um seine Theorie vor den Vertretern der Akademie definitiv ad absurdum $z u$ führen und unschädlich zu machen, läßt er es weder an scharfem Tadel ${ }^{32}$ ) noch an satirischen Anspielungen auf sein Pseudonym, von dem er mehr das Adjektivum verdiene als den Namen des Mannes, dessen Stelle er vertrat, - denn nur auf die Unfertigkeit und

31) Parm. $127 \mathrm{c}$.

,32) $135 \mathrm{~cd}$. 
Unreife der inneren Entwicklung des Speusippos, nicht auf die Zahl seiner Jahre beziehen sich jene ihn mit so auffallender Betonung als бழ̣óôp véov bezeichnenden Worte, deren scheinbare Harmlosigkeit dadurch eine schneidende Kritik enthält: denn sie machen aus dem an sich so ehrenvollen Pseudonym eine bittere Ironie, die um so tiefer treffen mußte, als diese geistige veóins mit dem Alter des etwa Vierzigjührigen (er ist mit Dion ungefähr gleichaltrig gewesen) genug kontrastierte -, fehlen: und mit dem Eingeständnis, nun in der Philosophie nicht mehr aus noch ein zu wissen, ${ }^{33}$ ) muß er, der als ein in "schülerhafter Haltungslosigkeit" von einer Hypothese zur andern seine Zuflucht nehmender Spekulant erscheint, schließlich den Meister um die ihm mangelnde Aufklärung bitten; die ihm für seinen Eifer zuteil gewordene Anerkennung ${ }^{34}$ ) hat dem gegenüber etwas so Ironisches, daß man mit dem Gelobten fast Mitleid hat: Platon hat die durch seinen Neffen heraufbeschworene Situation in ihrer ganzen Tragweite für die Zukunft so ernst genommen, als sie es nur verdiente.

Dem Aristoteles auf der andern Seite gibt er zu verstehen, wie nichtssagend jene angeblich „grundstürzenden" Einwände für die wahre Ideenlehre sind, indem er sie selbst als etwas gegen die Auffassung des Speusippos allerdings ganz auf der Hand Liegendes aufnimmt, zugleich aber als über etwas Triviales und Verächtliches so rasch er kann darüber hinweggeht. So verhütet er zugleich, daß dem durch seine Erfolge sicherlich nicht geringen Stolze des jungen Mannes, dessen Name bei dieser Polemik gar nicht genannt wird, neuer Stoff zugefügt werde, ein vornehmes Überseben, das ebenso 13ãab, wo von dem Gegner des Speusippos als von einem ungenannten $\tau_{s}$ die Rede ist, und vor allem in der Art, wie er etwas sehr von oben herab aus zufälligen äußeren Motiven zum Gesprächsgenossen des Parmenides ausersehen wird, deutlichen Ausdruck findet, und ihm, der es verstehe, daß man ihn in ganz anderer W'eise ernst nähme, gewiß höchst überraschend kam. Und nicht zum geringsten zeigt endlich die ganze Form des Gespräches mit ihm, in der Platon auf die seinen Dialogen eigentümliche

33) $127 \mathrm{c} ; 130 \mathrm{e} ; 135 \mathrm{c}$.

34) Parm. 135d. 
IVecliselwirkung der P'ersonen, wodurch dieselben immerhin objektiv gleichwertiger mit ilım dastehen, verzichtet, mit welchem souveränen Selbstbewußtsein er allzukühne Schüler durch Schlag auf Schlag erfolgende Fragen, bei denen sie ibre frühere Redseligkeit ganz verliißt, zum Bewußtsein ihrer Nichtigkeit zu bringen versteht. Ein pikanter Humor aber liegt darin, daß gerade der Schüler, der dem Speusippos gegenüber die platonische Ideenlehre mit solcher Zuversicht abgetan hatte, nun gerade den ehemaligen Lehrer über ihre Inentbehrlichkeit belehren muß, wodurch er zugleich seine damalige N'egation aufs glänzendste widerruft, dabei läßt es Platon nicht an ernsten Warnungen fehlen, wenn er hofft, daß Aristoteles als der Jüngste am wenigsten „Vorwitz ${ }^{(35}$ ) würde treiben wollen und die hohen geistigen Anforderungen betont, welche das Verständnis seiner

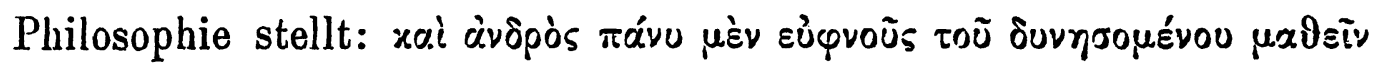

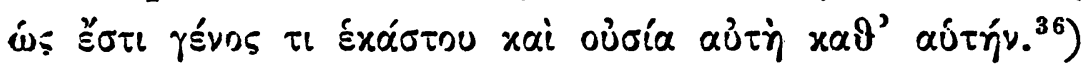

Das Gespräch war für noch wenigere geschrieben, als die wenigen dieses erlesenen Kreises, in denen wir, da sogar das Gros der Schule als die $\pi$ $\lesssim \lambda$ oí von ihnen abgesondert wird, nur die eigentlichen Lehrer und die Allerbevorzugtesten unter den Schülern der Akademie zu erkennen haben. ${ }^{37}$ ) Die Einigung, die Platon von der Sitzung erwartete, kam nicht zustande: im „Sophisten“ werden uns die beiden Parteien als die der Ideenfreunde und der gemäßigten Materialisten wieder begegnen. Aber die Verhandlungen dieser historischen Synusie vermachte Platon als Dokument in unserem Dialoge der Schule. Gewiß ist derselbe kein Stenogramm; die Sitzung wird wesentlich stürmischer verlaufen sein: auch diesmal hat der Philosoph ein Kunstwerk schaffen wollen. Aber sind es gewiß auch in seinen übrigen Dialogen "die gedanklichen Arbeiten der Akademie, seine Erfahrungen mit seinen Genossen und Schülern, deren bleibenden Gehalt er zu leuchtenden Gestalten hat kristallisieren lassen" ${ }^{38}$ ) so hat er doch keinem seiner Werke in solchem Grade selbst bis auf die Sprache den Charakter des Dokumenta-

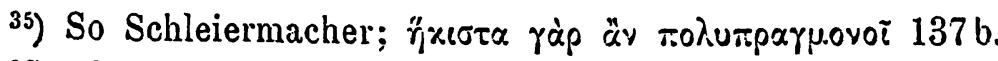

36) $135 \mathrm{a}$.

37) $136 \mathrm{~d}$.

38) Windelband, Platon ${ }^{3}$ p. 43.
} 
rischen, beinahe Protokollarischen gelassen, wie es dem Parmenides geschehen mußte gemäß seiner Bestimmung, für die Eingeweihten, welche die leichte Hülle durchschauten, jene definitive dialektische Begründung der Ideenlehre zu enthalten, wie sie mit Rücksicht auf eine historische Veranlassung an einem kritischen Tage der Akademie siegreich das Feld behauptet hatte.

Von diesem Gesichtspunkte aus betrachtet, erscheint die dreifache Wiedererzählung des Gespräches wie ein demselben absichtlich zu überzeugenderer Bestätigung dieser endgiltigen Darstellung dreimal aufgedrücktes Siegel, unter dessen Schutz die Reinheit der Lehre fürderhin gesichert sein soll; auch in dem von Überweg ${ }^{39}$ ) als geschmacklos verspotteten Umstand, daß Antiphon diesen Schuldialog auswendig gelernt hat, liegt, so persönlich auch diẹse Angabe sein mag, doch eine nicht mißzuverstehende Aufforderung an alle Platoniker ohne Ausnahme; die Personen, denen diese dreimalige Wiedererzählung in den Mund gelegt wird, sind natürlich zeitgenössische Akademiker; der Kephalos ist offenbar ein jetzt in Klazomenai wohnender ehemaliger Schüler Platons: mit welcher Einsicht wir allen chronologischen Spitzfindigkeiten glücklich überhoben sind. Geschrieben sein aber muß der Dialog in den letzten Monaten des Jabres 366, ehe sich noch der Eindruck des Ereignisses verwischt hatte.

Die wichtigste Folgerung eber ergibt sich aus unserer Analyse für das Verständnis von Platons Lehre. Da dieser Dialog als die fundamentale Darstellung der Ideenlehre besonders im Gegensatz zu der speusippischen transzendenten Fassung derselben von grundlegender Bedeutung für die Schule sein sollte, so haben wir in Zukunft kein Recht mehr, anstatt dem Platon zu geben, was ihm gehört, die aristotelische Darstellung jener Lehre; deren Irrtumsquelle nun aufgedeckt ist, als eine objektive anzuerkennen; vielmehr muß Platons Willen gemäß der "Parmenides" von nun an auch für uns der Markstein sein, von dem aus die Ideenlehre sowohl der früheren als der noch folgenden Dialoge allein begriffen werden darf.

39) Jabrb. für klass. Phil. 1863, IX, 110. 